\title{
Time of Development of Occlusal and Proximal Lesions: Implications for Fissure Sealants
}

\author{
Stephen A. Eklund, DDS, DrPH \\ Amid I. Ismail, BDS, DrPH* \\ Program in Dental Public Health \\ School of Public Health \\ The University of Michigan \\ Ann Arbor, Ml 48109-2029
}

\begin{abstract}
Analysis of surface and tooth-specific data from NHANES I and HHANES shows that the pattern of dental caries differs greatly from tooth to tooth and surface to surface. Occlusal caries precedes all other types, and increases most rapidly and to the highest levels in the molars. Caries on the proximal surfaces comes later.

For sealants to be of value, they must reduce the need for restorations. Therefore, the value of sealants will be determined by the balance between high enough levels of potential caries on occlusal surfaces to give them something to prevent, and low levels of, or sufficiently delayed, proximal lesions to prevent the loss of the otherwise saved occlusal surface.

The data presented demonstrate that as of the time of NHANES 1, the number of permanent molars that could have had surfaces saved from restoration, at least for a number of years, appears to be sufficient to warrant widespread use of sealants on those teeth. More recent data (HHANES) suggest that the current decline in caries prevalence is likeIy to reduce the value of sealants in the short run because there are fewer occlusal lesions in young children, but may increase the value of sealants in the long run because proximal caries in older children and adults is also declining.
\end{abstract}

Key Words: sealants, dental caries

\section{Introduction}

The adoption of dental sealants has been discussed widely, and it is often suggested that the recent decline in caries make the use of sealants an attractive option $(1,2)$. These suggestions are based primarily on the apparent decrease, because of fluorides, in the proportion of proximal surfaces that are carious. As fewer proximal surfaces require restoration, the occlusal surfaces that can be saved by sealants will not be lost in the process of proximal

\footnotetext{
*Now with the Department of Community Dentistry, Faculty of
} Dentistry, McGill University, Montreal, Quebec, H3A 2B2. restoration. This paper reviews available surfacespecific data to assess the implications for sealants of recent trends in dental caries.

\section{Available Data}

While there are numerous reports of DMFT and DMFS rates for specific subgroups, most notably 12-to-14-year-old children, there are few sources of data that provide tooth- and surface-specific caries data over a wide range of ages. Those data that do exist are usually limited to children, with the oldest group included rarely older than 18 years $(1,3,4)$. Exceptions in the last 15 years are the First $\mathrm{Na}$ tional Health and Nutrition Examination Survey (NHANES I) conducted from 1971 to 1974 and the Southwestern component of the Hispanic Health and Nutrition Examination Survey (HHANES), conducted from 1982 to 1984 , both under the direction of the National Center for Health Statistics (NCHS) $(5,6)$. Over 20,000 individuals ranging in age from one to 74 years were examined in NHANES I; 7,462 individuals aged six months to 74 years were examined in HHANES. The computer tapes with tooth and surface data from both surveys were obtained from NCHS for analysis. The data were compiled, surface by surface, tooth by tooth, for each single year age group in the surveys. Sampling-weight variables, estimated by NCHS, were used to correct for the oversampling used in the surveys. Detailed discussion of the sampling and its implications for analysis are presented elsewhere $(5,7)$.

These data from NHANES I and HHANES provide a picture of dental caries on a surface-by-surface basis in the respective populations at the time of each survey. Whereas the data from the Southwestern component of the HHANES do not, unlike NHANES I, represent a statistical sample of the United States population, the children in the sample are remarkably similar to all children from that region, based on comparisons with NIDR data from roughly the same time period (8).

It is also important to state that both are crosssectional surveys, and thus require cautious interpretation. It is impossible to say whether the pat- 
terns that appear in the older age groups in these data will be the same as the patterns that the younger groups will experience as they become older. On the other hand, it is hard to imagine that longitudinal data would be of any greater value, because by the time the decades required to gather them had passed (even if it were feasible to keep track of a group long enough to do so), the same concerns about applicability to today's children would remain.

\section{Tooth and Surface Data}

Occlusal surfaces. Critical questions about the magnitude of expected benefit from sealants concern which surfaces, and combinations of surfaces, can be expected to become carious in the absence of sealants, as well as the timing of these changes. For sealants to make any sense at all, the surfaces to which they will be applied must have some reasonable probability of becoming carious. Figure 1 presents age-specific data from NHANES I to show the percent of occlusal surfaces that were carious in the United States at that time. (The curves presented in Figures 1 to 6 have been slightly smoothed. The actual percentages for each age and surface are reported in Table 1. Note that Figure 1 groups the bicuspids in a slightly different manner than does Table 1.) The data demonstrate that for the occlusal surfaces of permanent molars at least 70 percent eventually become decayed or filled. This level is reached approximately 10 years after eruption. It is notable how much alike the patterns for the first and second molars are, except that the second molars are six years behind. The patterns for the occlusal surfaces from the HHANES data are remarkably

Figure 1

Decayed or Filled Occlusal Surfaces; Permanent Teeth: United States, 1971-74

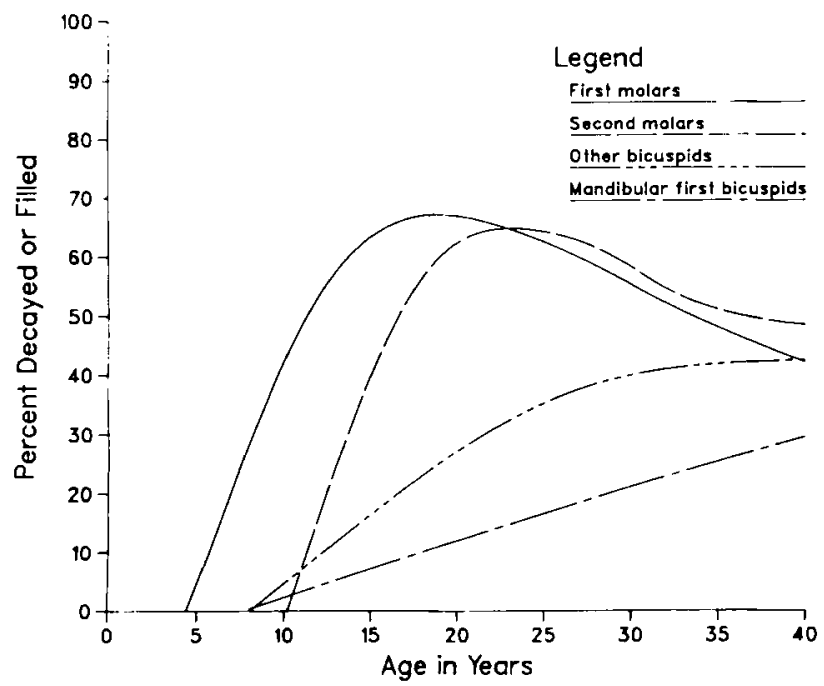

Source: National Center for Health Statistics: Special dental data tape from the Health and Nutrition Examination Survey, United States, 1971-74. similar, except that they are approximately 35 percent lower (Tables 1 and 2). Given the increasing missing component (not included in the graphs) in the older age ranges, the true percentage of teeth affected by occlusal caries is probably higher. Data beyond age 40 are not presented here because beyond that age the missing component of the index introduces a high degree of uncertainty.

The occlusal surfaces of the bicuspids present a different picture. For all but the mandibular first bicuspids, the bicuspids reach a peak in the area of 45 percent of occlusal surfaces carious or filled, and this peak is reached much later than in the molars, somewhere around age 30 . The different pattern is most apparent in the mandibular first bicuspids, where a lower peak, approximately 30 percent of occlusals carious or filled, is not reached until approximately 40 years of age. For the HHANES data, the pattern is similar; however, in this instance the values are approximately 45 percent lower than those in the NHANES I data (Tables 1 and 2).

Buccal and lingual surfaces. For the buccal surfaces of the mandibular molars (Figure 2), the pattern of caries and fillings is one of a slow rise to approximately 35 percent for the first molars and 20 percent for the second molars. These peaks are reached after age 20 for the first molars and at about 30 years of age for the second molars. For the lingual of the maxillary first molars, the peak of approximately 25 percent is reached around age 25 . For buccal and lingual surfaces on the molars, the HHANES data (Table 2) are the same or slightly higher than the equivalent data from NHANES I.

Proximal surfaces. From these data on pits and fissures, there appears to be ample reason to place

Figure 2

Decayed or Filled Surfaces; Selected Permanent Teeth and Surfaces: United States, 1971-74

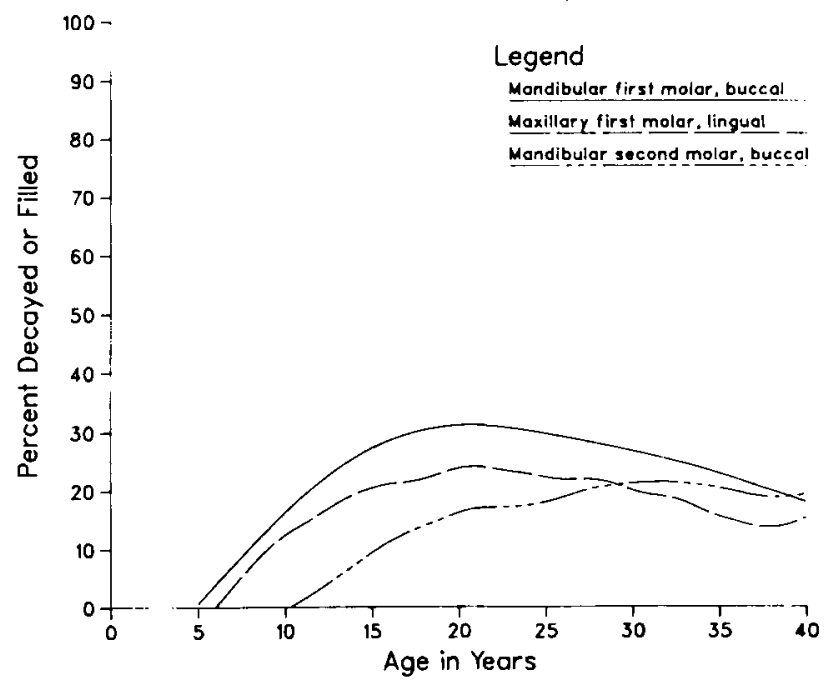

Source: National Center for Health Statistics: Special dental data tape from the Health and Nutrition Examination Survey, United States, 1971-74. 
TABLE 1

Percent of Selected Surfaces Decayed or Filled, Permanent Teeth: NHANES I (1971-74)

\begin{tabular}{|c|c|c|c|c|c|c|c|c|c|c|c|c|c|c|c|c|c|}
\hline \multirow[b]{3}{*}{ Age } & \multicolumn{17}{|c|}{ Percent Decayed or Filled Surfaces } \\
\hline & \multicolumn{4}{|c|}{ Occlusal } & \multicolumn{3}{|c|}{$\begin{array}{c}\text { Buccal or } \\
\text { Lingual }\end{array}$} & \multicolumn{4}{|c|}{ Proximal } & \multicolumn{2}{|c|}{$\begin{array}{l}\text { At Least } \\
\text { One } \\
\text { Proximal }\end{array}$} & \multicolumn{4}{|c|}{$\begin{array}{l}\text { Occlusal } \\
\text { With Sound } \\
\text { Proximals }\end{array}$} \\
\hline & $\mathrm{M} 2^{*}$ & M1 & B2 & B1 & M1L & $\mathrm{M} 1 \mathrm{~B}$ & M2B & M1M & M1D & $\mathrm{M} 2 \mathrm{M}$ & $\mathrm{M} 2 \mathrm{D}$ & M2 & M1 & M2 & M1 & B2 & B1 \\
\hline 5 & 0 & 1 & 0 & 0 & 0 & 0 & 0 & 0 & 0 & 0 & 0 & 0 & 0 & 0 & 1 & 0 & 0 \\
\hline 6 & 0 & 6 & 0 & 0 & 0 & 2 & 0 & 0 & 0 & 0 & 0 & 0 & 0 & 0 & 6 & 0 & 0 \\
\hline 7 & 0 & 12 & 0 & 0 & 3 & 6 & 0 & 0 & 0 & 0 & 0 & 0 & 1 & 0 & 11 & 0 & 0 \\
\hline 8 & 0 & 29 & 0 & 0 & 6 & 7 & 0 & 1 & 0 & 0 & 0 & 0 & 1 & 0 & 28 & 0 & 0 \\
\hline 9 & 0 & 40 & 2 & 1 & 12 & 18 & 0 & 3 & 2 & 0 & 0 & 0 & 4 & 0 & 36 & 1 & 1 \\
\hline 10 & 0 & 47 & 3 & 1 & 14 & 17 & 0 & 3 & 1 & 0 & 0 & 0 & 4 & 0 & 43 & 1 & 1 \\
\hline 11 & 2 & 50 & 3 & 2 & 12 & 20 & 1 & 6 & 3 & 0 & 0 & 0 & 7 & 2 & 42 & 2 & 2 \\
\hline 12 & 11 & 56 & 6 & 4 & 16 & 22 & 3 & 6 & 2 & 0 & 0 & 0 & 7 & 11 & 49 & 4 & 3 \\
\hline 13 & 25 & 62 & 10 & 6 & 18 & 23 & 4 & 12 & 5 & 0 & 2 & 1 & 14 & 24 & 48 & 7 & 4 \\
\hline 14 & 34 & 63 & 12 & 6 & 21 & 23 & 8 & 10 & 5 & 1 & 1 & 1 & 12 & 32 & 51 & 9 & 5 \\
\hline 15 & 37 & 62 & 13 & 9 & 19 & 33 & 9 & 10 & 5 & 2 & 2 & 3 & 12 & 35 & 50 & 8 & 5 \\
\hline 16 & 53 & 66 & 20 & 12 & 24 & 32 & 13 & 18 & 8 & 4 & 2 & 5 & 21 & 48 & 45 & 10 & 7 \\
\hline 17 & 56 & 65 & 21 & 16 & 20 & 26 & 14 & 19 & 11 & 5 & 4 & 7 & 22 & 49 & 43 & 10 & 8 \\
\hline 18 & 59 & 69 & 23 & 16 & 20 & 31 & 12 & 20 & 12 & 8 & 4 & 10 & 23 & 49 & 46 & 8 & 7 \\
\hline 19 & 59 & 66 & 24 & 15 & 24 & 29 & 14 & 23 & 13 & 6 & 3 & 7 & 27 & 51 & 39 & 10 & 8 \\
\hline 20 & 64 & 68 & 29 & 20 & 25 & 31 & 17 & 24 & 15 & 9 & 5 & 11 & 27 & 52 & 41 & 9 & 9 \\
\hline 21 & 66 & 65 & 30 & 25 & 25 & 37 & 21 & 26 & 19 & 12 & 7 & 14 & 31 & 52 & 34 & 10 & 9 \\
\hline 22 & 64 & 64 & 37 & 27 & 24 & 32 & 16 & 30 & 22 & 13 & 8 & 16 & 36 & 48 & 28 & 10 & 10 \\
\hline 23 & 63 & 64 & 32 & 22 & 21 & 28 & 17 & 25 & 19 & 11 & 7 & 14 & 31 & 48 & 33 & 9 & 7 \\
\hline 24 & 63 & 65 & 39 & 25 & 26 & 29 & 16 & 32 & 26 & 16 & 11 & 20 & 38 & 44 & 27 & 10 & 8 \\
\hline 25 & 62 & 62 & 41 & 28 & 21 & 29 & 18 & 30 & 21 & 14 & 7 & 15 & 36 & 47 & 27 & 9 & 8 \\
\hline 26 & 65 & 62 & 42 & 29 & 20 & 29 & 18 & 32 & 22 & 18 & 11 & 22 & 37 & 43 & 25 & 9 & 8 \\
\hline 27 & 62 & 59 & 46 & 34 & 23 & 27 & 21 & 32 & 27 & 19 & 11 & 22 & 37 & 40 & 22 & 9 & 8 \\
\hline 28 & 64 & 60 & 46 & 35 & 23 & 29 & 23 & 35 & 27 & 24 & 14 & 27 & 40 & 37 & 20 & 6 & 6 \\
\hline 29 & 63 & 58 & 43 & 31 & 23 & 27 & 19 & 31 & 25 & 23 & 12 & 26 & 35 & 37 & 23 & 9 & 6 \\
\hline 30 & 64 & 60 & 45 & 30 & 18 & 29 & 22 & 35 & 27 & 20 & 13 & 25 & 40 & 39 & 20 & 9 & 8 \\
\hline 31 & 51 & 47 & 38 & 29 & 17 & 24 & 20 & 29 & 22 & 20 & 13 & 25 & 32 & 26 & 15 & 8 & 8 \\
\hline 32 & 53 & 54 & 45 & 33 & 21 & 25 & 25 & 32 & 28 & 22 & 13 & 25 & 38 & 28 & 15 & 5 & 4 \\
\hline 33 & 48 & 46 & 38 & 34 & 21 & 23 & 18 & 28 & 26 & 19 & 14 & 22 & 33 & 26 & 12 & 6 & 6 \\
\hline 34 & 56 & 55 & 45 & 36 & 14 & 30 & 22 & 35 & 31 & 25 & 14 & 28 & 40 & 28 & 15 & 5 & 5 \\
\hline 35 & 50 & 47 & 40 & 32 & 15 & 24 & 22 & 27 & 23 & 23 & 14 & 25 & 31 & 25 & 16 & 4 & 6 \\
\hline 36 & 51 & 46 & 44 & 32 & 17 & 17 & 20 & 29 & 24 & 21 & 11 & 25 & 34 & 27 & 12 & 6 & 5 \\
\hline 37 & 49 & 48 & 39 & 36 & 13 & 22 & 18 & 32 & 27 & 22 & 16 & 26 & 36 & 22 & 12 & 5 & 6 \\
\hline 38 & 54 & 45 & 40 & 33 & 13 & 21 & 19 & 26 & 25 & 20 & 15 & 26 & 31 & 29 & 13 & 6 & 4 \\
\hline 39 & 42 & 37 & 41 & 29 & 13 & 17 & 16 & 29 & 21 & 17 & 11 & 20 & 28 & 23 & 9 & 4 & 4 \\
\hline 40 & 53 & 47 & 39 & 29 & 17 & 19 & 22 & 29 & 26 & 24 & 15 & 27 & 34 & 26 & 12 & 4 & 4 \\
\hline
\end{tabular}

* $\mathrm{M} 2$ stands for second molar; $M 1, M 2$, and B1 for first molar, second bicuspid, and first bicuspid, respectively. The suffixes $L, B, M$, and $D$ stand for lingual, buccal, mesial, and distal.

Source: National Center for Health Statistics: Special dental data tape from the First Health and Nutrition Examination Survey, United States, 1971-74.

sealants on all of these teeth. The nature of proximal restorations usually dictates, however, that the occlusal surface also be prepared and restored, whether it be carious or not. The probability of proximal lesions on these teeth, and the time of their appearance, is therefore also of importance to the value of sealants. Figure 3 shows the pattern for mesial and distal involvement in the permanent molars for the NHANES I data. Over 30 percent of the mesial surfaces and 30 percent of the distal surfaces in the first molars are carious in adults; a somewhat lower level is eventually reached in the second molars. Again, these may be underestimates, because of the growing percent of missing teeth into adulthood. An important factor to consider is that the proximal lesions appear later than 
TABLE 2

Percent of Selected Surfaces Decayed or Filled, Permanent Teeth: HHANES (1982-84)

\begin{tabular}{|c|c|c|c|c|c|c|c|c|c|c|c|c|c|}
\hline \multirow[b]{3}{*}{ Age } & \multicolumn{13}{|c|}{ Percent Decayed or Filled Surfaces } \\
\hline & \multicolumn{4}{|c|}{ Occlusal } & \multicolumn{3}{|c|}{$\begin{array}{l}\text { Buccal or } \\
\text { Lingual }\end{array}$} & \multicolumn{2}{|c|}{$\begin{array}{l}\text { At Least } \\
\text { One } \\
\text { Proximal }\end{array}$} & \multicolumn{4}{|c|}{$\begin{array}{c}\text { Occlusal } \\
\text { With Sound } \\
\text { Proximals }\end{array}$} \\
\hline & $\mathrm{M} 2^{*}$ & M1 & B2 & B1 & M1L & M1B & M2B & M2 & M1 & M2 & M1 & B2 & B1 \\
\hline 5 & 0 & 0 & 0 & 0 & 0 & 0 & 0 & 0 & 0 & 0 & 0 & 0 & 0 \\
\hline 6 & 0 & 3 & 0 & 0 & 1 & 2 & 0 & 0 & 0 & 0 & 3 & 0 & 0 \\
\hline 7 & 0 & 8 & 0 & 0 & 2 & 7 & 0 & 0 & 0 & 0 & 8 & 0 & 0 \\
\hline 8 & 0 & 14 & 0 & 0 & 6 & 17 & 0 & 0 & 0 & 0 & 14 & 0 & 0 \\
\hline 9 & 0 & 24 & 1 & 0 & 10 & 16 & 0 & 0 & 1 & 0 & 24 & 0 & 0 \\
\hline 10 & 1 & 28 & 1 & 1 & 11 & 24 & 0 & 0 & 2 & 1 & 26 & 0 & 0 \\
\hline 11 & 2 & 37 & 2 & 1 & 19 & 28 & 1 & 0 & 4 & 2 & 32 & 1 & 1 \\
\hline 12 & 7 & 41 & 2 & 1 & 18 & 32 & 7 & 0 & 4 & 7 & 37 & 1 & 0 \\
\hline 13 & 17 & 49 & 4 & 3 & 25 & 40 & 11 & 0 & 7 & 17 & 42 & 2 & 2 \\
\hline 14 & 16 & 42 & 4 & 3 & 20 & 30 & 9 & 0 & 5 & 15 & 37 & 2 & 2 \\
\hline 15 & 29 & 50 & 6 & 5 & 32 & 40 & 15 & 1 & 9 & 28 & 42 & 4 & 4 \\
\hline 16 & 30 & 51 & 8 & 5 & 28 & 40 & 15 & 1 & 10 & 30 & 42 & 4 & 3 \\
\hline 17 & 38 & 58 & 11 & 6 & 37 & 44 & 19 & 2 & 13 & 35 & 44 & 7 & 4 \\
\hline 18 & 34 & 43 & 11 & 6 & 24 & 37 & 20 & 3 & 10 & 31 & 33 & 6 & 4 \\
\hline 19 & 37 & 47 & 12 & 8 & 22 & 36 & 15 & 3 & 13 & 34 & 34 & 5 & 4 \\
\hline 20 & 37 & 42 & 15 & 9 & 28 & 33 & 21 & 3 & 13 & 34 & 29 & 7 & 5 \\
\hline 21 & 40 & 43 & 18 & 11 & 23 & 32 & 20 & 5 & 14 & 35 & 29 & 8 & 5 \\
\hline 22 & 42 & 43 & 18 & 10 & 22 & 31 & 18 & 6 & 16 & 36 & 27 & 6 & 4 \\
\hline 23 & 42 & 42 & 15 & 7 & 22 & 38 & 21 & 6 & 14 & 36 & 28 & 8 & 3 \\
\hline 24 & 54 & 57 & 24 & 12 & 31 & 43 & 27 & 9 & 22 & 45 & 36 & 6 & 3 \\
\hline 25 & 44 & 45 & 24 & 13 & 26 & 33 & 20 & 5 & 18 & 39 & 27 & 10 & 6 \\
\hline 26 & 46 & 40 & 21 & 13 & 24 & 27 & 23 & 10 & 17 & 36 & 23 & 7 & 6 \\
\hline 27 & 41 & 39 & 18 & 13 & 19 & 21 & 20 & 8 & 14 & 33 & 26 & 7 & 7 \\
\hline 28 & 43 & 44 & 23 & 16 & 24 & 30 & 17 & 9 & 21 & 34 & 24 & 5 & 4 \\
\hline 29 & 46 & 43 & 24 & 13 & 20 & 32 & 19 & 8 & 18 & 38 & 25 & 7 & 5 \\
\hline 30 & 44 & 39 & 18 & 13 & 23 & 28 & 28 & 9 & 16 & 35 & 23 & 5 & 4 \\
\hline 31 & 46 & 40 & 20 & 14 & 21 & 26 & 25 & 8 & 15 & 38 & 25 & 7 & 4 \\
\hline 32 & 40 & 39 & 23 & 17 & 20 & 21 & 20 & 11 & 18 & 28 & 21 & 5 & 4 \\
\hline 33 & 48 & 36 & 24 & 13 & 17 & 27 & 18 & 11 & 19 & 37 & 17 & 6 & 3 \\
\hline 34 & 48 & 44 & 26 & 19 & 22 & 26 & 18 & 14 & 21 & 34 & 23 & 7 & 6 \\
\hline 35 & 43 & 42 & 26 & 22 & 19 & 28 & 18 & 12 & 21 & 31 & 21 & 6 & 8 \\
\hline 36 & 44 & 38 & 32 & 22 & 25 & 25 & 22 & 17 & 23 & 27 & 15 & 8 & 8 \\
\hline 37 & 44 & 36 & 26 & 15 & 17 & 20 & 18 & 8 & 19 & 35 & 18 & 7 & 4 \\
\hline 38 & 51 & 41 & 32 & 23 & 19 & 28 & 28 & 19 & 23 & 32 & 18 & 6 & 8 \\
\hline 39 & 41 & 34 & 28 & 22 & 14 & 21 & 20 & 11 & 18 & 30 & 16 & 7 & 5 \\
\hline 40 & 45 & 39 & 25 & 19 & 12 & 23 & 21 & 11 & 19 & 34 & 20 & 9 & 6 \\
\hline
\end{tabular}

* $\mathrm{M}$ stands for second molar; M1, B2, and B1 for first molar, second bicuspid, and first bicuspid, respectively. The suffixes L, B, M, and D stand for lingual, buccal, mesial, and distal.

Source: National Center for Health Statistics: Dental data tape from the Southwestern Component of the Hispanic Health and Nutrition Examination Survey, 1982-84.

the occlusal lesions. This pattern suggests that those looking at ratios of lesions by surface type only in children may seriously underestimate the importance of proximal caries. Figure 4 demonstrates this phenomenon for the molars. The two upper curves show that the percent of molars with occlusal caries or fillings rises sharply soon after eruption. The two lower lines, representing the percent of teeth with at least one proximal surface with a filling or carious lesion, show a notably different trend.

The HHANES data for the proximal surfaces (Table 2) again shows a pattern like that in NHANES I, but in this instance is more than 50 percent lower 
Figure 3

Decayed or Filled Proximal Surfaces; Permanent Molars: United States, 1971-74

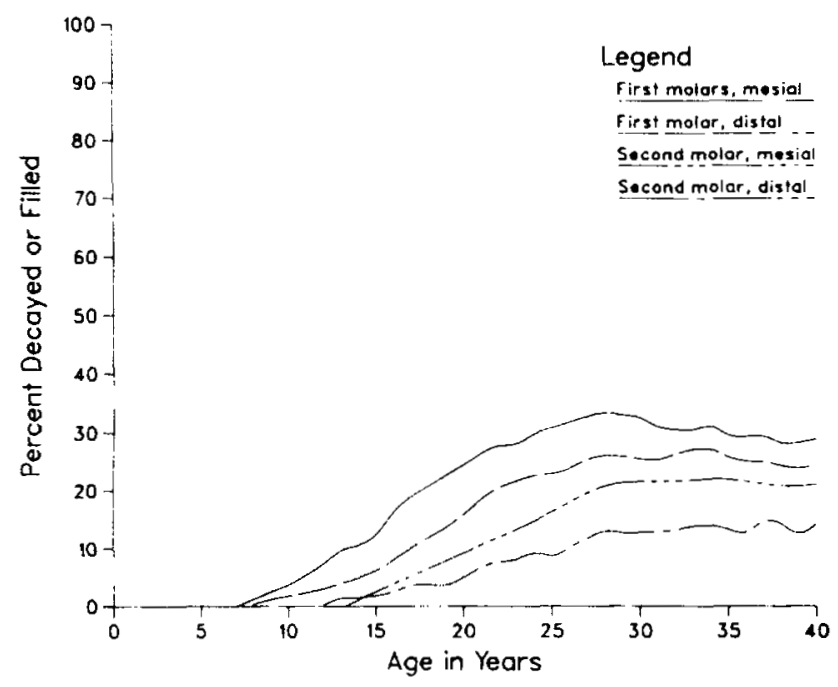

Source: National Center for Health Statistics: Special dental data tape from the Health and Nutrition Examination Survey, United States, 1971-74.

throughout the entire range from five to 40 years of age.

Combinations of surfaces. Possibly the most important data are provided by subtracting the proximal surface data from the data for the occlusal surfaces. Figure 5 shows the percent of the various tooth types from NHANES I that have filled or decayed occlusals, but have both proximal surfaces sound. In one sense these teeth can be characterized as the potential "successes" with respect to sealants. The occlusal is carious, but the proximals are not. An important thing to notice is that the patterns for molars are remarkably different from the patterns for the bicuspids. Molars eventually achieve a level of around 50 percent in this category-that is, occlusal caries or fillings could have been potentially avoided in at least half of all molar teeth. The similarity of the patterns of first and second molars, except for the six-year offset, is again evident. The bicuspids, on the other hand, never get appreciably above 10 percent. So while sealants have the potential for preventing the need for occlusal fillings in 50 percent of the molars, sealants can do no better than to save the need for occlusal fillings in 10 percent of the bicuspids.

Another important characteristic is the apparent rapid drop-off in the percent of these potential "successes" that appears approximately 10 years after eruption. The source of this drop-off is important. Until approximately 30 years of age, the majority of this drop-off is because of proximal lesions. In other words, many of the occlusal surfaces that could have been saved by occlusal sealants would eventually be filled because of the need for proximal restorations. Sealing of buccal and lingual pits
Figure 4

Decayed or Filled Surfaces; Permanent Molars: United States, 1971-74

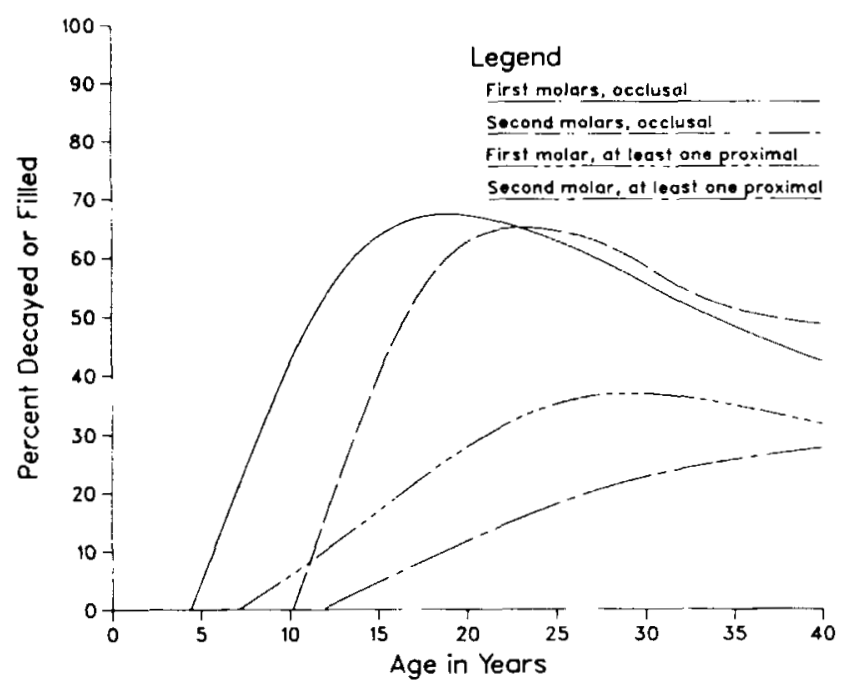

Source: National Center for Health Statistics: Special dental data tape from the Health and Nutrition Examination Survey, United States, 1971-74.

Figure 5

Decayed or Filled Occlusals with Sound Proximals; Permanent Teeth: United States, 1971-74

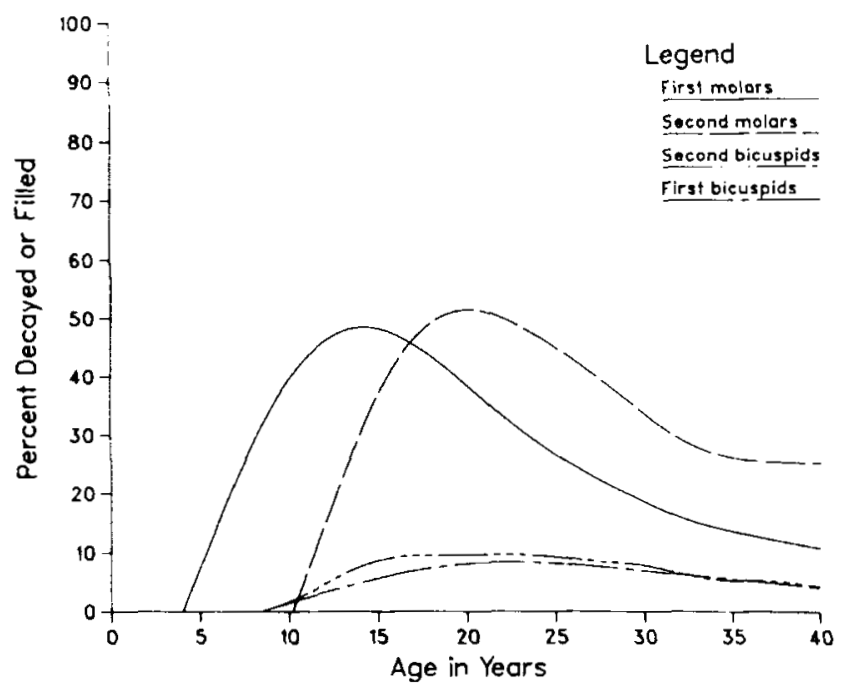

Source: National Center for Health Statistics: Special dental data tape from the Health and Nutrition Examination Survey, United States, 1971-74.

of molars is, of course, not subject to later loss because of proximal caries. Beyond age 30, a growing majority of the drop-off is attributable in the NHANES I data to tooth loss, and therefore, with these data, is impossible to characterize with precision.

In contrast to the data from NHANES I, the data in Table 2 from HHANES show a consistent pattern of lower prevalence for both occlusal and proximal 
Figure 6

Decayed or Filled Occlusals with Sound Proximals; Permanent Molars: United States 1971-74 and Hispanic Population of Southwestern United States, 1982-84

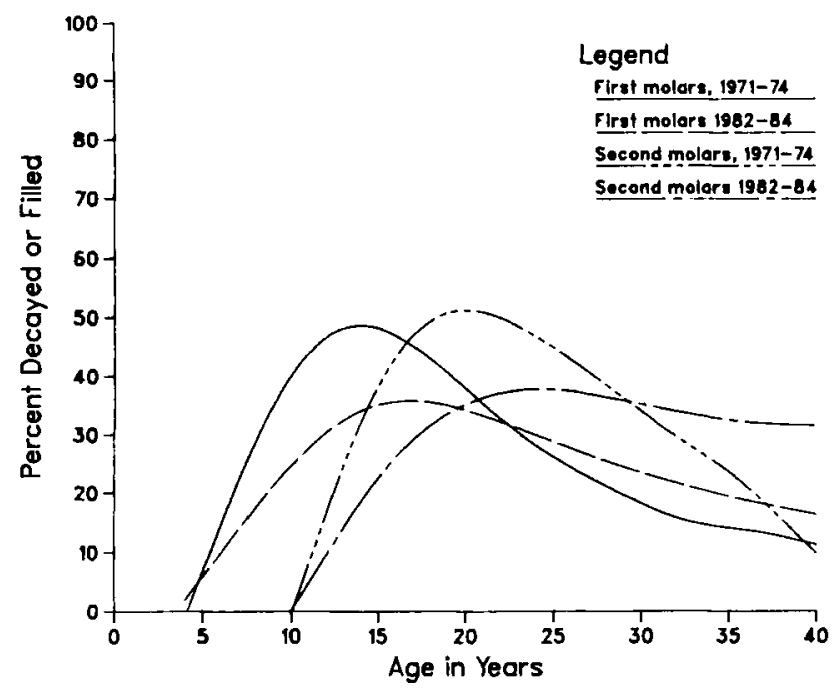

Source: National Center for Health Statistics. The 1971-74 data are from the First Health and Nutrition Examination Survey. The 1982-84 data are from the Southwestern Component of the Hispanic Health and Nutrition Examination Survey.

surfaces. While the populations from which the data come are not the same and therefore should not be compared directly, the fact that Hispanic children from HHANES are remarkably similar to all children from the same region at roughly the same time (8) suggests that the caries patterns from HHANES are a plausible representation of the patterns to be expected in lower-prevalence populations.

Figure 6 combines the curves for the potential "successes" for first and second molars from the NHANES (1971-74) data with those from the HHANES (1982-84). The combined data in Figure 6 demonstrate that for approximately 15 or 20 years following tooth eruption there are fewer potential sealant "successes" in the lower-prevalence population than there were in 1971-74.

The main reason for the reduced number of potential successes in the first two decades after eruption in the lower-prevalence population is the smaller number of occlusal lesions and fillings in childhood. While there is also a considerable reduction in proximal lesions and fillings at the same ages, the absolute numbers involved are smaller; therefore, the occlusal changes are the primary reason for the difference in potential successes between the two groups. By early adulthood, however, this pattern appears to change. The reduced prevalence of proximal lesions begins to overcome the reduced prevalence of occlusal lesions and fillings. This combination, together with fewer missing teeth (see Table 3 ), produce a net increase in
TABLE 3

Percent of Permanent Teeth Missing, United States, 1971-74, and Hispanic Population from the Southwestern United States, 1982-84

\begin{tabular}{rrrrrr}
\hline & \multicolumn{2}{c}{ NHANES I } & 1971-74 & & \multicolumn{2}{c}{ HHanes } & 1982-84 \\
\cline { 2 - 3 } \cline { 5 - 6 } Age & M2* & M1 & & M2 & M1 \\
\hline 5 & 0 & 0 & & 0 \\
10 & 0 & 3 & & 0 \\
15 & 3 & 12 & & 1 \\
20 & 6 & 12 & & 5 & 8 \\
25 & 16 & 25 & & 11 \\
30 & 23 & 31 & & 5 & 14 \\
35 & 40 & 45 & & 15 & 18 \\
40 & 34 & 41 & & 14 & 22
\end{tabular}

* $\mathrm{M} 2$ and $\mathrm{M} 1$ stand for second and first molars, respectively.

potential sealant "successes" in adults in the lower prevalence population.

\section{Discussion}

The usual concern with the use of cross-sectional data to characterize a disease is that if disease levels are changing, the pattern revealed may not properly represent the actual disease experience of any particular age group. In the instance of a declining disease, the levels in the older age groups will not be reached by those in the younger ages as they get older and the levels in the younger groups are lower than the older group experienced when they were young. While longitudinal data are clearly superior for characterizing the pattern experienced by a specific group, these data too may be of limited value in understanding past or future patterns. In the circumstance of changing disease levels, it is not at all clear that longitudinal data are superior to cross-sectional data for forecasting future patterns of disease. Potential uncertainties come not from the type of the data, but from the fact that caries rates are changing. The real challenge then, regardless of the type of data, is to use the available data with appropriate caution regarding the likely effect of changes in disease patterns.

The data presented from NHANES I suggest patterns of development of dental caries that appear to be highly favorable for the use of fissure sealants. Occlusal caries in the permanent molars occurs many years in advance of proximal caries, and occurs in a high enough percentage of children to warrant a serious preventive effort. Even if some of the "saved" occlusal surfaces are lost later in life because of restorations to proximal lesions, these surfaces still represent restorations prevented because only one restoration to the occlusal surface instead of two is required.

It has been argued that the recent caries reduction makes the picture for sealants even more fa- 
vorable (1). The reasoning behind this assertion is that the major source of the recent caries decline is a decline in proximal caries. The data presented, however, reveal that to accomplish reductions in caries of the magnitude reported since the time of the NHANES I survey-36 percent in the National Caries Prevalence Survey (4)-the majority of the reduction (in absolute terms) must have come from pit-and-fissure caries, because children at the time of NHANES I simply did not have many proximal lesions. For example, in 12-year-old children, only seven percent of first molars had one or more proximal lesions, while 56 percent of the same teeth had carious or filled occlusals. Clearly, a caries decline of the magnitude reported in the last few years must be partially attributed to a decline in occlusal caries. The difference in potential sealant "success$\mathrm{es}^{\prime \prime}$ in children between NHANES I and HHANES is, in fact, largely attributable to lower prevalence in occlusal caries. This phenomenon has been noted by several groups of researchers. Stamm stated that "[in the Netherlands] over the period from 1969 to 1978, it has been the decline in occlusal fissure caries that has accounted for the largest absolute drop in decay" (9). Reduction in the range of 30 percent in the occlusal surfaces of molars between NHANES I and the National Caries Prevalence Survey have been reported by Swango and Brunelle (10). Ripa and coworkers noted that ". . . occlusal surfaces [exhibited] the highest reduction ... after a fluoride mouthrinse program (11).

\section{"So, while sealants have the potential for preventing the need for occlusal fill- ings in 50 percent of the molars, seal- ants can do no better than to save the need for occlusal fillings in 10 percent of the bicuspids."}

It has been noted already that the caries prevalence in HHANES is markedly lower than in NHANES I on occlusal and proximal surfaces. An unexpected finding is that filled or carious buccal surfaces on mandibular molars and lingual surfaces on maxillary molars are at least as prevalent in HHANES as they were in NHANES I. If this finding is valid, that is, if buccal-and-lingual-pit lesions have reached some sort of "lower limit" below which they will not fall despite declining prevalence on other surfaces, it will be of great importance to the potential value of sealants. The reader is cautioned, however, not to accept this finding without reservation. Perhaps the most plausible explanation is that because far fewer teeth are lost to extraction in HHANES than in NHANES I (Table $3)$, that more of the carious and filled buccal and lingual surfaces are still available for scoring. If one assumes that a high percentage of extracted mo- lars in children and young adults had a filled or carious buccal or lingual pit, then the prevalence for those surfaces could, in fact, actually be lower in HHANES than NHANES I.

While adequate data from young adults presently are not available, the available data do suggest that because of the current decline in caries prevalence, the potential "successes" curve will be lower in children because there will be fewer occlusal lesions to prevent. If the current caries decline is at least partially a phenomenon of delay as opposed to true primary prevention as described by Jackson (12), the curve will climb with posteruptive age, but at a slower rate. In addition, if the caries reduction also applies to the proximal caries that is first diagnosed in adulthood, the curve will not exhibit the abrupt falloff seen in the NHANES I data. The net result could be an apparent reduction (relative to NHANES I) of the potential for sealants in childhood, but an enhancement of their ultimate value when the child reaches adulthood-very much like the pattern in Figure 6, which compares NHANES I with HHANES. The likely net effect of the recent reduction in dental caries is, therefore, to reduce the number of sealant "successes" in the short run, because fewer occlusal lesions are occurring in young children, and to increase the number of "successes" in the long run because of a likely reduction of proximal caries in older children and adults.

\section{Conclusions}

1. At the time of the NHANES I survey, occlusal caries or restorations could have been prevented by sealants in at least 50 percent of all permanent molar teeth. For bicuspids, occlusal caries or restorations could have been prevented in approximately 10 percent of the teeth.

2. In comparing U.S. children in 1971-74 (NHANES I) with a lower-prevalence population (HHANES, 1982-84), the greatest absolute difference in caries is in occlusal lesions and restorations.

3 . If sealants were to come into widespread use, the likely net effect of the recent reduction in dental caries would be to reduce the number of sealant "successes" in the short run because fewer occlusal lesions are occurring in young children, but to increase the number of "successes" in the long run because of a likely reduction of proximal caries in older children and adults.

\section{Acknowledgments}

The authors thank Ms. Mary Dudley and Mr. Jerry Wheeler of the National Center for Health Statistics for their help and cooperation in providing NHANES I and HHANES data.

\section{References}

1. Bohannan HM, et al. Indications for sealant use in a community-based preventive dentistry program. J Dent Educ 1984 Feb;48(Suppl):45-55. 
2. Burt BA. Cost-effectiveness of sealants in private practice and standards for use in prepaid care. J Am Dent Assoc 1985 Jan;110:103-7.

3. Klein H, Palmer CE. Studies on dental caries. XII. Comparison of the caries susceptibility of the various morphological types of permanent teeth. J Dent Res 1941 June;20:203-16.

4. US Public Health Service, National Institute of Dental Research, National Caries Program. The prevalence of dental caries in United States children, 1979-1980. 1981 NIH Publ No 82-2245 Washington DC; Government Printing Office, iv +159 p.

5. National Center for Health Statistics: Plan and operation of the Health and Nutrition Examination Survey, United States, 1971-1973, by H Miller. Vital and Health Statistics. Series 1 Nos. 10a and 10b. DHEW Pub No (HSM) 73-1310. Health Services and Mental Health Administration. Washington DC; Government Printing Office, Feb 1973.

6. National Center of Health Statistics: Plan and operation of the Hispanic Health and Health and Nutrition Examination Survey, United States, 1982-84, Vital and Health Statistics. Series 1 . In preparation. Draft available from the authors.
7. Landis JR, Lepkowski JM, Eklund SA, Stehouwer SA. A statistical methodology for analyzing data from a complex survey: the first National Health and Nutrition Examination Survey. Hyattsville, MD: National Center for Health Statistics, 1982; DHHS publication no. (PHS) 82-1366.

8. Ismail AI, Burt BA. Prevalence of dental caries and periodontal disease in Southwestern Hispanic children aged five to 17 years. Results from Southwestern HHANES, 1982-84. Submitted for publication, 1985.

9. Stamm JW. Is there a need for dental sealants?: Epidemiological indications in the 1980's. J Dent Educ, 1984 Feb;48 (Suppl):9-17.

10. Swango PA, Brunelle JA. Age- and surface-specific caries attack rates from the National Dental Caries Prevalence Survey. [Abstract]. J Dent Res 1983 Mar;62(Spec Iss):270.

11. Ripa LW, Leske GS, Sposato A. Surface-specific caries pattern of participants in a school-based fluoride mouthrinsing program with implications for the use of sealants. J Public Health Dent 1985 Spring; 45:90-4.

12. Jackson D. Dental caries: the distinction between delay and prevention. Br Dent J 1974 Nov;137:347-51.

\section{Americans are Sweet on Sugar}

The US Department of Agriculture estimates that Americans consumed an average of 71 pounds of sucrose per person in 1983, down about 32 pounds since 1972. That may seem to be good news, but since then the use of corn sweeteners has more than doubled. And over the next several years, average yearly high fructose corn syrup intake alone is expected to level off at about 39 pounds per person. Indeed, the consumption of caloric sweeteners seems to be staying fairly constant-about 125 pounds for each person, but that's equivalent to more than 600 calories per person each day! [Recent studies show] that sugar is not the direct cause of obesity, diabetes, or heart disease [and] there is little if any well-founded support for claims that sugar is responsible for hyperactivity of any psychological problems. Nevertheless, it is well substantiated that sugars-particularly caloric sweeteners - contribute to tooth decay and provide little nutritional value. How much sugar, then, is acceptable? The US Dietary Goals suggest that refined sugars should constitute no more than 10 percent of the total calories you eat a day-that means 200 calories for a person who needs 2,000 calories a day.

-in Tufts University Diet $\mathcal{E}$ Nutrition Letter 3(3):3-6, 